Commun. Fac. Sci. Univ. Ank. Ser. A1 Math. Stat.

Volume 68, Number 2, Pages 1359-1369(2019)

DOI: $10.31801 /$ cfsuasmas.530441

ISSN 1303-5991 E-ISSN 2618-6470

http://communications.science.ankara.edu.tr/index.php?series=A1

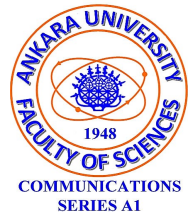

\title{
ON GENERALIZED TAXICAB METRIC IN THREE DIMENSIONAL SPACE
}

\author{
HARUN BARIŞ ÇOLAKOĞLU
}

\begin{abstract}
In this paper, we define the generalized taxicab distance function in three dimensional space, which includes the taxicab distance function as a special case, and we show that three dimensional generalized taxicab distance function determines a metric. Then we give some properties of three dimensional generalized taxicab metric, and determine Euclidean isometries of the space preserving the generalized taxicab metric.
\end{abstract}

\section{INTRODUCTION}

Taxicab geometry was introduced by Menger [15, and developed by Krause [13, using the taxicab metric which is the special case of the well-known $l_{p}$-metric (also known as Minkowski distance) for $p=1$. In [18, Lawrance J. Wallen altered the taxicab metric by redefining in order to get rid of imperative symmetry, and called it the (slightly) generalized taxicab metric. During the recent years, metric geometries based on these metrics have been studied and developed in many directions. See [1, [2], 4], [5], 6], 7], 9, [10, 11], [12, 16], and [17] for some of studies.

In this work, we define the generalized taxicab distance function in three dimensional space, and we show that three dimensional generalized taxicab distance function determines a metric in $\mathbb{R}^{3}$, that is, $d_{T_{g}}$ is a function from $\mathbb{R}^{3} \times \mathbb{R}^{3}$ to $[0, \infty)$ satisfying the following conditions: (1) $d_{T_{g}}(A, B)=0$ if and only if $A=B$, (2) $d_{T_{g}}(A, B)=d_{T_{g}}(B, A)$, and $(3) d_{T_{g}}(A, B) \leq d_{T_{g}}(A, C)+d_{T_{g}}(C, B)$ for all $A$, $B$, and $C$ in $\mathbb{R}^{3}$. Then we give some properties of three dimensional generalized taxicab metric, and determine Euclidean isometries of the space which preserve the generalized taxicab metric.

\section{The generalized taxicab Distance in $\mathbb{R}^{3}$}

The generalized taxicab distance between two points in $\mathbb{R}^{3}$ can be defined as follows:

Received by the editors: March 08, 2017, Accepted: August 10, 2018.

2010 Mathematics Subject Classification. 51K05, 51K99, 51N99.

Key words and phrases. Metric, taxicab distance, generalized taxicab distance, isometry.

Com 2019 Ankara University
Commications Faculty of Sciences University of Ankara-Series A1 Mathematics and Statistics 
Definition 1. Let $A=\left(x_{1}, y_{1}, z_{1}\right)$ and $B=\left(x_{2}, y_{2}, z_{2}\right)$ be two points in $\mathbb{R}^{3}$. For each positive real numbers $a, b$, and $c$, the function $d_{T_{g}}: \mathbb{R}^{3} \times \mathbb{R}^{3} \rightarrow[0, \infty)$ defined by

$$
d_{T_{g}}(A, B)=a\left|x_{1}-x_{2}\right|+b\left|y_{1}-y_{2}\right|+c\left|z_{1}-z_{2}\right|
$$

is called generalized taxicab distance function in $\mathbb{R}^{3}$, and the real number $d_{T_{q}}(A, B)$ is called generalized taxicab distance between points $A$ and $B$. As a special case, if $a=b=c=1$, then

$$
d_{T_{g}}(A, B)=d_{T}(A, B)=\left|x_{1}-x_{2}\right|+\left|y_{1}-y_{2}\right|+\left|z_{1}-z_{2}\right|
$$

which is the well-known taxicab distance in $\mathbb{R}^{3}$.

Remark 2. We are quite familiar to the notation of $d_{T}$ for the taxicab distance function. In [5] and [6], $d_{T_{g}}$ was used to state the generalized taxicab distance. One can find the definition of $d_{T_{g}}$ not to be well-defined since the generalized taxicab distance between two points can change according to values $a, b$, and $c$. To remove this confusion, we have to use values $a, b$ and $c$ in the name of the distance. This can be done easily; for example by using the notation $(a, b, c)$ in phrase of $d_{T_{g}}$. But we keep on using $d_{T_{g}}$ for the sake of shortness, supposing values $a, b$ and $c$ are initially determined and fixed unless otherwise stated.

The following proposition shows that the generalized taxicab distance function satisfies the metric properties in $\mathbb{R}^{3}$.

Proposition 3. The generalized taxicab distance function determines a metric in $\mathbb{R}^{3}$.

Proof. Clearly, $d_{T_{g}}(A, B)=0$ if and only if $A=B$, and $d_{T_{g}}(A, B)=d_{T_{g}}(B, A)$ for any points $A$ and $B$ in $\mathbb{R}^{3}$. So, we only will show that $d_{T_{g}}$ satisfies the triangle inequality, that is, $d_{T_{g}}(A, B) \leq d_{T_{g}}(A, C)+d_{T_{g}}(C, B)$ for points $A=\left(x_{1}, y_{1}, z_{1}\right)$, $B=\left(x_{2}, y_{2}, z_{2}\right)$, and $C=\left(x_{3}, y_{3}, z_{3}\right)$ in $\mathbb{R}^{3}$. This fact can be proven as follows:

$$
\begin{aligned}
& d_{T_{g}}(A, B)=a\left|x_{1}-x_{2}\right|+b\left|y_{1}-y_{2}\right|+c\left|z_{1}-z_{2}\right| \\
& =a\left|\left(x_{1}-x_{3}\right)+\left(x_{3}-x_{2}\right)\right|+b\left|\left(y_{1}-y_{3}\right)+\left(y_{3}-y_{2}\right)\right|+c\left|\left(z_{1}-z_{3}\right)+\left(z_{3}-z_{2}\right)\right| \\
& \leq a\left|x_{1}-x_{3}\right|+b\left|y_{1}-y_{3}\right|+c\left|z_{1}-z_{3}\right|+a\left|x_{3}-x_{2}\right|+b\left|y_{3}-y_{2}\right|+c\left|z_{3}-z_{2}\right| \\
& =d_{T_{g}}(A, C)+d_{T_{g}}(C, B) .
\end{aligned}
$$

\section{Some properties of $d_{T_{g}}$ IN $\mathbb{R}^{3}$}

Let $A=\left(x_{1}, y_{1}, z_{1}\right)$ and $B=\left(x_{2}, y_{2}, z_{2}\right)$ be two points in the three dimensional Cartesian coordinate space. Let $\Psi_{X}^{x}, \Psi_{X}^{y}$, and $\Psi_{X}^{z}$ be planes through the point $X$ and perpendicular to the $x$-axis, $y$-axis and $z$-axis, respectively. Since the Euclidean distances from $A$ to planes $\Psi_{B}^{x}, \Psi_{B}^{y}$, and $\Psi_{B}^{z}$ (or from the point $B$ to planes $\Psi_{A}^{x}$, $\Psi_{A}^{y}$, and $\left.\Psi_{A}^{z}\right)$ are $d_{E}\left(A, \Psi_{B}^{x}\right)=\left|x_{1}-x_{2}\right|, d_{E}\left(A, \Psi_{B}^{y}\right)=\left|y_{1}-y_{2}\right|$ and $d_{E}\left(A, \Psi_{B}^{z}\right)=$ $\left|z_{1}-z_{2}\right|$, the generalized taxicab distance between points $A$ and $B$ can be given by

$$
d_{T_{g}}(A, B)=a d_{E}\left(A, \Psi_{B}^{x}\right)+b d_{E}\left(A, \Psi_{B}^{y}\right)+c d_{E}\left(A, \Psi_{B}^{z}\right) .
$$



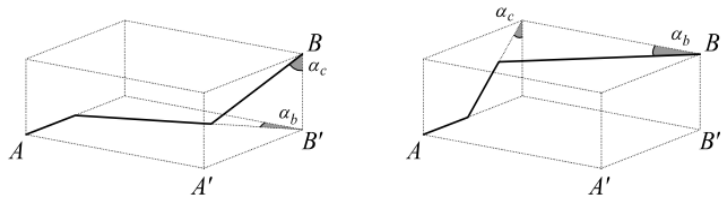

$\max \{a, b, c\}=a$
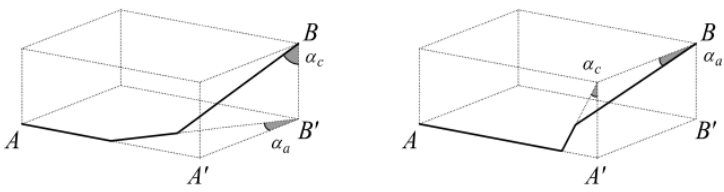

$\max \{a, b, c\}=b$
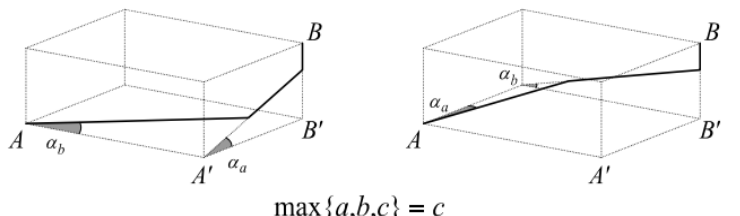

Figure 1. Some shortest paths from $A$ to $B$.

Clearly, one can consider the generalized taxicab metric as a generalization of the $\alpha$ metric in three dimensional space (see [ $[\underline{6}$ and $[3]$ ). So, for $i \in\{a, b, c\}$, let us define $\frac{x}{\max \{a, b, c\}}=\left(\sec \alpha_{i}-\tan \alpha_{i}\right)$ such that $\alpha_{i} \in[0, \pi / 2)$, to determine the shortest paths from $A$ to $B$. Thus, the generalized taxicab distance between points $A$ and $B$ is constant $\max \{a, b, c\}$ multiple of the Euclidean length of one of shortest paths from $A$ and $B$ composed of line segments making angles $\alpha_{a}, \alpha_{b}$ and $\alpha_{c}$ with $x$, $y$ and $z$ axes respectively, as in Figure 1. As a special case, if $a=b=c$, then $\alpha_{a}=\alpha_{b}=\alpha_{c}=0$, and the generalized taxicab distance between points $A$ and $B$ is constant $a$ multiple of the Euclidean length of one of the shortest paths from $A$ to $B$ composed of line segments, each parallel to one of planes $\Psi_{B}^{x}, \Psi_{B}^{y}$, and $\Psi_{B}^{z}$ : That is, $d_{T_{g}}(A, B)=a d_{T}(A, B)$.

There exist, in general, infinitely many shortest paths between points $A$ and $B$. For $\max \{a, b, c\}=a$, if $A$ and $B$ are not at the same plane, then all possible cases for shapes of shortest paths from $A$ to $B$ are shown in Figure 2.

For points $A=\left(x_{1}, y_{1}, z_{1}\right)$ and $B=\left(x_{2}, y_{2}, z_{2}\right)$ in $\mathbb{R}^{3}$, we denote by $R_{A B}$ the rectangular prismatic region or rectangular region or a line segment bounded by planes $\Psi_{A}^{x}, \Psi_{A}^{y}, \Psi_{A}^{z}, \Psi_{B}^{x}, \Psi_{B}^{y}$, and $\Psi_{B}^{z}$. The following corollary follow directly from Eq. (3) which is the geometric interpretation of the generalized taxicab distance.

Corollary 4. Let $A, B$ and $C$ be three points in $\mathbb{R}^{3}$ such that $C \in R_{A B}$. Then, $d_{T_{g}}(A, B) \geq d_{T_{g}}(A, C)$. In addition, $d_{T_{g}}(A, B)=d_{T_{g}}(A, C)$ if and only if $B=C$. 

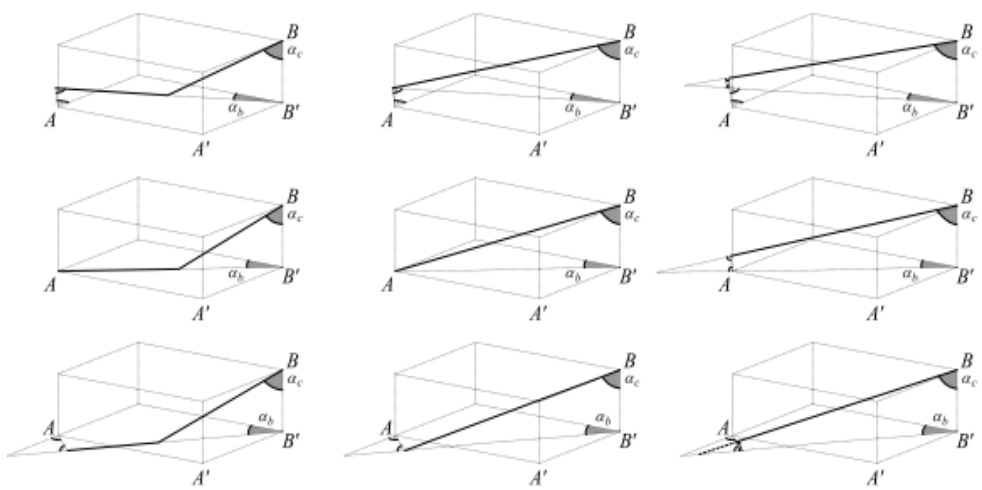

Figure 2. Possible shortest paths for $\max \{a, b, c\}=a$.

$d_{T_{g}}(A, B)=a\left(\left|x_{1}-x_{2}\right|+\left(\sec \alpha_{b}-\tan \alpha_{b}\right)\left|y_{1}-y_{2}\right|+\left(\sec \alpha_{c}-\tan \alpha_{c}\right)\left|z_{1}-z_{2}\right|\right)$

Let us denote the space endowed with the generalized taxicab metric by $\mathbb{R}_{T_{g}}^{3}$. Then unit generalized taxicab sphere in $\mathbb{R}_{T_{g}}^{3}$ is the set of points $(x, y, z)$ in the space satisfying the equation $a|x|+b|y|+c|z|=1$. One can see by calculation that unit generalized taxicab sphere is an octahedron with vertices $A_{1}=\left(\frac{1}{a}, 0,0\right)$, $A_{2}=\left(\frac{-1}{a}, 0,0\right), A_{3}=\left(0, \frac{1}{b}, 0\right), A_{4}=\left(0, \frac{-1}{b}, 0\right), A_{5}=\left(0,0, \frac{1}{c}\right)$, and $A_{6}=\left(0,0, \frac{-1}{c}\right)$ (see Figure 3). If $a=b=c$, then unit generalized taxicab sphere is a regular octahedron.

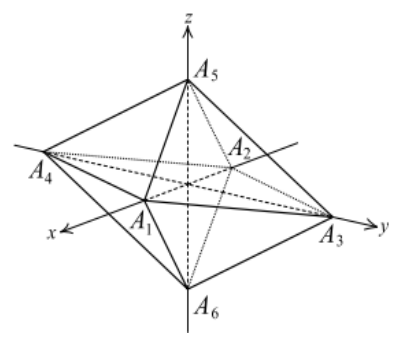

Figure 3. Unit generalized taxicab sphere for $a=1 / 2, b=1 / 7$, $c=1 / 4$.

The following proposition gives an equation which relates the Euclidean distance to the generalized taxicab distance between two points in the Cartesian coordinate space:

Proposition 5. For any two points $A$ and $B$ in $\mathbb{R}^{3}$, if $(p, q, r)$ is the direction vector of the line through $A$ and $B$, then

$$
d_{E}(A, B)=\rho(p, q, r) d_{T_{g}}(A, B)
$$


where $\rho(p, q, r)=\left(p^{2}+q^{2}+r^{2}\right)^{1 / 2} /(a|p|+b|q|+c|r|)$.

Proof. Let $A=\left(x_{1}, y_{1}, z_{1}\right)$ and $B=\left(x_{2}, y_{2}, z_{2}\right)$ such that $(p, q, r)$ is the direction vector of the line through $A$ and $B$. Then $x_{1}-x_{2}=\lambda p, y_{1}-y_{2}=\lambda q$ and $z_{1}-z_{2}=\lambda r$ for $\lambda \in \mathbb{R}$. Now, one can derive by a straightforward calculation that $d_{E}(A, B) / d_{T_{g}}(A, B)=\left(p^{2}+q^{2}+r^{2}\right)^{1 / 2} /(a|p|+b|q|+c|r|)$, thus we have Eq. (4).

The following two corollaries follow directly from Proposition 5:

Corollary 6. Let $A, B, C$ and $D$ be four points in $\mathbb{R}^{3}$. If lines $A B$ and $C D$ are coincident or parallel, then

$$
d_{T_{g}}(A, B)=d_{T_{g}}(C, D) \text { if and only if } d_{E}(A, B)=d_{E}(C, D) .
$$

If $A, B$ and $X$ are three distinct collinear points in $\mathbb{R}^{3}$, then

$$
d_{T_{g}}(A, X) / d_{T_{g}}(X, B)=d_{E}(A, X) / d_{E}(X, B) .
$$

As a consequence of Corollary 6 and Corollary 7, it is clear that Thales, Menelaus, and Ceva theorems are true in $\mathbb{R}_{T_{g}}^{3}$.

\section{Euclidean isometries Which PReserve $d_{T_{g}}$}

An isometry is a transformation of the space onto itself which preserves distances. One of the basic geometric problems for a given metric space is to determine the isometries of it. It is well-known that the group of isometries of the Euclidean space with $d_{E}$ metric is $G=E(3)$, which is the semidirect product of the translation group $T(3)$ consisting of all translations of the space, and the symmetry group of unit sphere $O(3)$. In the remaining part of this study, we will determine Euclidean isometries of $\mathbb{R}_{T_{g}}^{3}$ which preserve the generalized taxicab distance, with Euclidean notions of translation, inversion, reflection, rotation, rotary reflection and rotary inversion, as in 7 .

Definitions which adopted from Martin [14] as follows: If $\Delta$ is a plane, then the reflection $\sigma_{\Delta}$ is the mapping on the points in $\mathbb{R}_{T_{g}}^{3}$ such that $\sigma_{\Delta}(X)=X$ if the point $X$ is on the plane $\Delta$, and $\sigma_{\Delta}(X)=Y$ if the point $X$ is not on the plane $\Delta$, and the plane $\Delta$ is the perpendicular bisector of the line segment $X Y$. If two planes $\Gamma$ and $\Delta$ intersect at a line $l$, then $\sigma_{\Delta} \sigma_{\Gamma}$ is called a rotation about the axis $l$. If two planes $\Gamma$ and $\Delta$ are parallel, then $\sigma_{\Delta} \sigma_{\Gamma}$ is called a translation. If $\Gamma$ and $\Delta$ are two intersecting planes each perpendicular to plane $\Pi$, then $\sigma_{\Pi} \sigma_{\Delta} \sigma_{\Gamma}$ is called a rotary reflection about the point common to $\Gamma, \Delta$ and $\Pi$. If $M$ is a point, the inversion (or point reflection) $\sigma_{M}$ about $M$ is the transformation such that $\sigma_{M}(X)=Y$ for all points $X$ where $M$ is the midpoint of $X$ and $Y$. If $\Gamma$ and $\Delta$ are two intersecting planes and $M$ is a point common to $\Gamma$ and $\Delta$, then $\sigma_{M} \sigma_{\Delta} \sigma_{\Gamma}$ is called a rotary inversion. 
It is obvious that all translations $\tau_{\left(x_{0}, y_{0}, z_{0}\right)}(x, y, z)=\left(x+x_{0}, y+y_{0}, z+z_{0}\right)$ and the inversion about the origin $\sigma_{O}(x, y, z)=(-x,-y,-z)$ preserve the generalized taxicab distance, by Eq. (1) or Corollary 6. So, they are isometries of $\mathbb{R}_{T_{g}}^{3}$. Note that if a Euclidean isometric transformation fixing the origin do not fix the generalized taxicab sphere, then it is not a generalized taxicab isometry. So, to show a Euclidean isometric transformation not to be a generalized taxicab isometry, one can simply control the images of vertices of the unit generalized taxicab sphere in Figure 3 with the following properties, under these transformations:

$$
\begin{aligned}
& \text { if } a=b=c \text {, then }\left|O A_{1}\right|_{E}=\left|O A_{2}\right|_{E}=\left|O A_{3}\right|_{E}=\left|O A_{4}\right|_{E}=\left|O A_{5}\right|_{E}=\left|O A_{6}\right|_{E}, \\
& \text { if } a=b \neq c \text {, then }\left|O A_{1}\right|_{E}=\left|O A_{2}\right|_{E}=\left|O A_{3}\right|_{E}=\left|O A_{4}\right|_{E} \neq\left|O A_{5}\right|_{E}=\left|O A_{6}\right|_{E} \text {, } \\
& \text { if } a=c \neq b \text {, then }\left|O A_{1}\right|_{E}=\left|O A_{2}\right|_{E}=\left|O A_{5}\right|_{E}=\left|O A_{6}\right|_{E} \neq\left|O A_{3}\right|_{E}=\left|O A_{4}\right|_{E} \text {, }
\end{aligned}
$$

if $a \neq b=c$, then $\left|O A_{1}\right|_{E}=\left|O A_{2}\right|_{E} \neq\left|O A_{3}\right|_{E}=\left|O A_{4}\right|_{E}=\left|O A_{5}\right|_{E}=\left|O A_{6}\right|_{E}$, if $a \neq b \neq c \neq a$, then $\left|O A_{1}\right|_{E}=\left|O A_{2}\right|_{E} \neq\left|O A_{3}\right|_{E}=\left|O A_{4}\right|_{E} \neq\left|O A_{5}\right|_{E}=$ $\left|O A_{6}\right|_{E} \neq\left|O A_{1}\right|_{E}$.

If the image of a vertex of the unit generalized taxicab sphere under a Euclidean isometric transformation fixing the origin, is not a vertex of the unit generalized taxicab sphere, then it is not a generalized taxicab isometry.

Clearly, if $a=b=c$, then $d_{T_{g}}\left(P_{1}, P_{2}\right)=a d_{T}\left(P_{1}, P_{2}\right)$ for any two points $P_{1}=$ $\left(x_{1}, y_{1}, z_{1}\right)$ and $P_{2}=\left(x_{2}, y_{2}, z_{2}\right)$ in $\mathbb{R}_{T_{g}}^{3}$. So, Euclidean isometries which preserve the generalized taxicab distance are the same as Euclidean isometries of the taxicab space $\mathbb{R}_{T}^{3}$ determined in [7]. The following four propositions determine Euclidean isometries of $\mathbb{R}_{T_{g}}^{3}$ which preserve the generalized taxicab distance for $a=b=c$. Here, we use coordinate definition of a reflection about a plane through the origin and the matrix representation of a rotation about a line passing through the origin to determine the image of $(x, y, z)$ under these transformations (see [7, pp. 191,193).

Proposition 7. Assume that $a=b=c$, then a reflection about a plane through the origin is an isometry if and only if the plane is perpendicular to one of vectors in the set

$$
D=\{(1,0,0),(0,1,0),(0,0,1),(1, \pm 1,0),(1,0, \pm 1),(0,1, \pm 1)\} .
$$

Thus, there exist only nine reflections about planes through the origin that preserve the generalized taxicab distance.

Proof. Let $a=b=c$, and let $\sigma_{(p, q, r)}$ denote the reflection about a plane through the origin and perpendicular to the vector $(p, q, r)$. Then, one can see that 
$\sigma_{(1,0,0)}(x, y, z)=(-x, y, z), \sigma_{(0,1,0)}(x, y, z)=(x,-y, z), \sigma_{(0,0,1)}(x, y, z)=(x, y,-z)$,

$\sigma_{(1,1,0)}(x, y, z)=(-y,-x, z), \sigma_{(1,-1,0)}(x, y, z)=(y, x, z)$,

$\sigma_{(1,0,1)}(x, y, z)=(-z, y,-x), \sigma_{(1,0,-1)}(x, y, z)=(z, y, x)$,

$\sigma_{(0,1,1)}(x, y, z)=(x,-z,-y), \sigma_{(0,1,-1)}(x, y, z)=(x, z, y)$.

So, if $\sigma_{(p, q, r)}\left(P_{i}\right)=P_{i}^{\prime}$ for $(p, q, r) \in D$, then $d_{T_{g}}\left(P_{1}, P_{2}\right)=d_{T_{g}}\left(P_{1}^{\prime}, P_{2}^{\prime}\right)$. Since the other reflections about a plane through the origin do not fix the generalized taxicab sphere, they are not isometry.

Proposition 8. Assume that $a=b=c$, then a rotation $r_{(p, q, r), \theta}$ about a line passing through the origin with the direction vector $(p, q, r)$, is an isometry if and only if $r_{(p, q, r), \theta} \in R_{1} \cup R_{2} \cup R_{3}$ such that

$R_{1}=\left\{r_{(p, q, r), \theta}: \theta \in\{\pi / 2, \pi, 3 \pi / 2\}\right.$ and $\left.(p, q, r) \in D_{1}\right\}$

$R_{2}=\left\{r_{(p, q, r), \theta}: \theta \in\{2 \pi / 3,4 \pi / 3\}\right.$ and $\left.(p, q, r) \in D_{2}\right\}$

$R_{3}=\left\{r_{(p, q, r), \theta}: \theta=\pi\right.$ and $\left.(p, q, r) \in D_{3}\right\}$, where

$D_{1}=\{(1,0,0),(0,1,0),(0,0,1)\}$

$D_{2}=\{(1,1,1),(-1,1,1),(1,-1,1),(1,1,-1)\}$ and

$D_{3}=\{(1,1,0),(1,0,1),(0,1,1),(1,-1,0),(1,0,-1),(0,1,-1)\}$.

Thus, there exist only twenty-three rotations about lines through origin that preserve the generalized taxicab distance.

Proof. Let $a=b=c$. Using the matrix representation of $r_{(p, q, r), \theta}$ one gets

$r_{(1,0,0), \frac{\pi}{2}}(x, y, z)=(x, z,-y), r_{(1,0,0), \pi}(x, y, z)=(x,-y,-z), r_{(1,0,0), \frac{3 \pi}{2}}(x, y, z)=(x,-z, y)$,

$r_{(0,1,0), \frac{\pi}{2}}(x, y, z)=(-z, y, x), r_{(0,1,0), \pi}(x, y, z)=(-x, y,-z), r_{(0,1,0), \frac{3 \pi}{2}}(x, y, z)=(z, y,-x)$,

$r_{(0,0,1), \frac{\pi}{2}}(x, y, z)=(y,-x, z), r_{(0,0,1), \pi}(x, y, z)=(-x,-y, z), r_{(0,0,1), \frac{3 \pi}{2}}(x, y, z)=(-y, x, z)$,

$r_{(1,1,1), \frac{2 \pi}{3}}(x, y, z)=(y, z, x), r_{(1,1,1), \frac{4 \pi}{3}}(x, y, z)=(z, x, y)$,

$r_{(-1,1,1), \frac{2 \pi}{3}}(x, y, z)=(-z,-x, y), r_{(-1,1,1), \frac{4 \pi}{3}}(x, y, z)=(-y, z,-x)$,

$r_{(1,-1,1), \frac{2 \pi}{3}}(x, y, z)=(z,-x,-y), r_{(1,-1,1), \frac{4 \pi}{3}}(x, y, z)=(-y,-z, x)$,

$r_{(1,1,-1), \frac{2 \pi}{3}}(x, y, z)=(-z, x,-y), r_{(1,1,-1), \frac{4 \pi}{3}}(x, y, z)=(y,-z,-x)$,

$r_{(1,1,0), \pi}(x, y, z)=(y, x,-z), r_{(1,-1,0), \pi}(x, y, z)=(-y,-x,-z)$,

$r_{(1,0,1), \pi}(x, y, z)=(z,-y, x), r_{(1,0,-1), \pi}(x, y, z)=(-z,-y,-x)$,

$r_{(0,1,1), \pi}(x, y, z)=(-x, z, y), r_{(0,1,-1), \pi}(x, y, z)=(-x,-z,-y)$.

So, if $r_{(p, q, r), \theta}\left(P_{i}\right)=P_{i}^{\prime}$ for $\theta \in\{\pi / 2, \pi, 3 \pi / 2\}$ and $(p, q, r) \in D_{1}$, or $\theta \in\{2 \pi / 3,4 \pi / 3\}$ and $(p, q, r) \in D_{2}$, or $\theta=\pi$ and $(p, q, r) \in D_{3}$, then $d_{T_{g}}\left(P_{1}, P_{2}\right)=d_{T_{g}}\left(P_{1}^{\prime}, P_{2}^{\prime}\right)$. Since the other rotations about a line passing through the origin do not fix the generalized taxicab sphere, they are not isometry.

Proposition 9. Assume that $a=b=c$, then a rotary reflection $\sigma_{\Pi} \sigma_{\Delta} \sigma_{\Gamma}=$ $\sigma_{(p, q, r)} r_{(p, q, r), \theta}$ about origin $(\Pi \cap \Delta \cap \Gamma=O)$ is an isometry if and only if $\theta \in$ $\{\pi / 2,3 \pi / 2\}$ and rotation axis has a direction vector $(p, q, r) \in D_{1}$, and $\Pi$ is the plane through origin and perpendicular to the axis of rotation. Thus, there exist only six rotary reflections about origin that preserve the generalized taxicab distance. 
Proof. Let $a=b=c$. Using coordinate definitions one can find that

$\sigma_{(1,0,0)} r_{(1,0,0), \frac{\pi}{2}}(x, y, z)=(-x, z,-y), \sigma_{(1,0,0)} r_{(1,0,0), \frac{3 \pi}{2}}(x, y, z)=(-x,-z, y)$,

$\sigma_{(0,1,0)} r_{(0,1,0), \frac{\pi}{2}}(x, y, z)=(-z,-y, x), \sigma_{(0,1,0)} r_{(0,1,0), \frac{3 \pi}{2}}(x, y, z)=(z,-y,-x)$,

$\sigma_{(0,0,1)} r_{(0,0,1), \frac{\pi}{2}}(x, y, z)=(y,-x,-z), \sigma_{(0,0,1)} r_{(0,0,1), \frac{3 \pi}{2}}(x, y, z)=(-y, x,-z)$.

So, if $\sigma_{(p, q, r)} r_{(p, q, r), \theta}\left(P_{i}\right)=P_{i}^{\prime}$ for $\theta \in\{\pi / 2,3 \pi / 2\}$ and $(p, q, r) \in D_{1}$, then

$d_{T_{g}}\left(P_{1}, P_{2}\right)=d_{T_{g}}\left(P_{1}^{\prime}, P_{2}^{\prime}\right)$. Since any transformation such as $\sigma_{(p, q, r)} r_{(p, q, r), \theta}$ different from the generalized taxicab isometric transformations determined before, does not fix the generalized taxicab sphere, it is not an isometry.

Proposition 10. Assume that $a=b=c$, then a rotary inversion $\sigma_{O} \sigma_{\Delta} \sigma_{\Gamma}=$ $\sigma_{O} r_{(p, q, r), \theta}$ about origin $(O \in \Delta \cap \Gamma)$ is an isometry if and only if $\theta \in\{2 \pi / 3,4 \pi / 3\}$ and rotation axis has a direction vector $(p, q, r) \in D_{2}$. Thus, there exist only eight rotary inversions about origin that preserve the generalized taxicab distance.

Proof. Let $a=b=c$. One can control following transformations

$\sigma_{O} r_{(1,1,1), \frac{2 \pi}{3}}(x, y, z)=(-y,-z,-x), \sigma_{O} r_{(1,1,1), \frac{4 \pi}{3}}(x, y, z)=(-z,-x,-y)$,

$\sigma_{O} r_{(-1,1,1), \frac{2 \pi}{3}}(x, y, z)=(z, x,-y), \sigma_{O} r_{(-1,1,1), \frac{4 \pi}{3}}(x, y, z)=(y,-z, x)$,

$\sigma_{O} r_{(1,-1,1), \frac{2 \pi}{3}}(x, y, z)=(-z, x, y), \sigma_{O} r_{(1,-1,1), \frac{4 \pi}{3}}(x, y, z)=(y, z,-x)$,

$\sigma_{O} r_{(1,1,-1), \frac{2 \pi}{3}}(x, y, z)=(z,-x, y), \sigma_{O} r_{(1,1,-1), \frac{4 \pi}{3}}(x, y, z)=(-y, z, x)$.

So, if $\sigma_{O} r_{(p, q, r), \theta}\left(P_{i}\right)=P_{i}^{\prime}$ in which $\theta \in\{2 \pi / 3,4 \pi / 3\}$ and $(p, q, r) \in D_{2}$, then $d_{T_{g}}\left(P_{1}, P_{2}\right) \neq d_{T_{g}}\left(P_{1}^{\prime}, P_{2}^{\prime}\right)$ for every $P_{1}, P_{2}$ in $\mathbb{R}_{T_{g}}^{3}$. Since any transformation such as $\sigma_{O} r_{(p, q, r), \theta}$ different from the generalized taxicab isometric transformations determined before, does not fix the generalized taxicab sphere, it is not an isometry.

Note that we did not take account of any rotary reflection or rotary inversion which is identical to a generalized taxicab isometric transformations that we determined before. So, pay attention to the following identical transformations: If $(p, q, r) \in D_{1}$ then $\sigma_{(p, q, r)} r_{(p, q, r), \pi}=\sigma_{O}, \sigma_{O} r_{(p, q, r), \frac{\pi}{2}}=\sigma_{(p, q, r)} r_{(p, q, r), \frac{3 \pi}{2}}$, $\sigma_{O} r_{(p, q, r), \frac{3 \pi}{2}}=\sigma_{(p, q, r)} r_{(p, q, r), \frac{\pi}{2}}$, and if $(p, q, r) \in D_{1} \cup D_{3}$, then $\sigma_{O} r_{(p, q, r), \pi}=\sigma_{(p, q, r)}$.

Now, for the cases $a=b \neq c, a=c \neq b, a \neq b=c$ and $a \neq b \neq c \neq a$, to determine Euclidean isometries of $\mathbb{R}_{T_{g}}^{3}$ which preserve the generalized taxicab distance let us define sets $D_{a}=\{(1,0,0)\}, D_{b}=\{(0,1,0)\}, D_{c}=\{(0,0,1)\}, D_{a, b}=$ $\{(1,1,0),(1,-1,0)\}, D_{a, c}=\{(1,0,1),(1,0,-1)\}$, and $D_{b, c}=\{(0,1,1),(0,1,-1)\}$. In the following propositions' proofs will be given only for the case $a=b \neq c$. Other cases can be done similarly, and left to the reader.

Proposition 11. A reflection about a plane through the origin is an isometry if the plane is perpendicular to one of vectors in the set

$$
D^{\prime}= \begin{cases}D_{1} \cup D_{x, y} & , \text { if } x=y \neq z \text { where } x, y, z \in\{a, b, c\} \\ D_{1} & \text { if } a \neq b \neq c \neq a\end{cases}
$$


Thus, if $a=b \neq c, a=c \neq b$ or $a \neq b=c$, there exist only five, and if $a \neq b \neq c \neq a$ there exist only three reflections about planes through the origin that preserve the generalized taxicab distance.

Proof. Let $a=b \neq c$. Then, one can see that

$\sigma_{(1,0,0)}(x, y, z)=(-x, y, z), \sigma_{(0,1,0)}(x, y, z)=(x,-y, z), \sigma_{(0,0,1)}(x, y, z)=(x, y,-z)$, $\sigma_{(1,1,0)}(x, y, z)=(-y,-x, z), \sigma_{(1,-1,0)}(x, y, z)=(y, x, z)$.

So, if $\sigma_{(p, q, r)}\left(P_{i}\right)=P_{i}^{\prime}$ for $(p, q, r) \in D_{1} \cup D_{a, b}$, then $d_{T_{g}}\left(P_{1}, P_{2}\right)=d_{T_{g}}\left(P_{1}^{\prime}, P_{2}^{\prime}\right)$. Since the other reflections do not fix the generalized taxicab sphere, they are not isometry, for the case $a=b \neq c$.

Proposition 12. A rotation $r_{(p, q, r), \theta}$ about a line $l$ passing through the origin and with direction vector $(p, q, r)$, is an isometry if and only if $r_{(p, q, r), \theta} \in R^{\prime}$ such that

$R^{\prime}=\left\{\begin{array}{cl}\left\{r_{(p, q, r), \theta}: \theta \in\{\pi / 2,3 \pi / 2\} \text { and }(p, q, r) \in D_{z}\right. & \\ \left.\text { or } \theta=\pi \text { and }(p, q, r) \in D_{1} \cup D_{x, y}\right\}, & \text { if } x=y \neq z ; x, y, z \in\{a, b, c\} \\ \left\{r_{(p, q, r), \theta}: \theta=\pi \text { and }(p, q, r) \in D_{1}\right\}, & \text { if } a \neq b \neq c \neq a\end{array}\right.$

Thus, if $a=b \neq c, a=c \neq b$ or $a \neq b=c$, there exist only seven, and if $a \neq b \neq c \neq a$ there exist only three rotations about lines through the origin that preserve the generalized taxicab distance.

Proof. Let $a=b \neq c$. Then, we see that

$r_{(0,0,1), \frac{\pi}{2}}(x, y, z)=(y,-x, z), r_{(0,0,1), \frac{3 \pi}{2}}(x, y, z)=(-y, x, z)$,

$r_{(1,0,0), \pi}(x, y, z)=(x,-y,-z), r_{(0,1,0), \pi}(x, y, z)=(-x, y,-z), r_{(0,0,1), \pi}(x, y, z)=$ $(-x,-y, z)$

$r_{(1,1,0), \pi}(x, y, z)=(y, x,-z), r_{(1,-1,0), \pi}(x, y, z)=(-y,-x,-z)$.

So, if $r_{(p, q, r), \theta}\left(P_{i}\right)=P_{i}^{\prime}$ for $\theta \in\{\pi / 2, \pi, 3 \pi / 2\}$ and $(p, q, r) \in D_{c}$, or $\theta=\pi$ and $(p, q, r) \in D_{1} \cup D_{a, b}$, then $d_{T_{g}}\left(P_{1}, P_{2}\right)=d_{T_{g}}\left(P_{1}^{\prime}, P_{2}^{\prime}\right)$. Since the other rotations do not fix the generalized taxicab sphere, they are not isometry, for the case $a=b \neq c$.

Proposition 13. If $x=y \neq z$ for $x, y, z \in\{a, b, c\}$, a rotary reflection $\sigma_{\Pi} \sigma_{\Delta} \sigma_{\Gamma}=$ $\sigma_{(p, q, r)} r_{(p, q, r), \theta}$ about the origin is an isometry if and only if $\theta \in\{\pi / 2,3 \pi / 2\}$ and $(p, q, r) \in D_{z}$, and $\Pi$ is the plane through the origin and perpendicular to $(p, q, r)$. Thus, if $a=b \neq c, a=c \neq b$ or $a \neq b=c$, there exist only two rotary reflections about the origin that preserve the generalized taxicab distance. If $a \neq b \neq c \neq a$, there is no rotary reflection that preserve the generalized taxicab distance, different from the generalized taxicab isometric transformations determined before.

Proof. Let $a=b \neq c$. We know that

$\sigma_{(0,0,1)} r_{(0,0,1), \frac{\pi}{2}}(x, y, z)=(y,-x,-z), \sigma_{(0,0,1)} r_{(0,0,1), \frac{3 \pi}{2}}(x, y, z)=(x, y,-z)$.

So, if $\sigma_{(p, q, r)} r_{(p, q, r), \theta}\left(P_{i}\right)=P_{i}^{\prime}$ in which $\theta \in\{\pi / 2,3 \pi / 2\}$ and $(p, q, r) \in D_{c}$, then $d_{T_{g}}\left(P_{1}, P_{2}\right)=d_{T_{g}}\left(P_{1}^{\prime}, P_{2}^{\prime}\right)$. Since any transformation such as $\sigma_{(p, q, r)} r_{(p, q, r), \theta}$ different from the generalized taxicab isometric transformations determined before, does not fix the generalized taxicab sphere, it is not an isometry, for the case $a=b \neq c$. 
Proposition 14. If $x=y \neq z$ for $x, y, z \in\{a, b, c\}$ or $a \neq b \neq c \neq a$, there is no rotary inversion $\sigma_{O} \sigma_{\Delta} \sigma_{\Gamma}=\sigma_{O} r_{(p, q, r), \theta}$ about the origin that preserve the generalized taxicab distance, different from the generalized taxicab isometric transformations determined before.

Proof. Let $a=b \neq c$. One can see that any transformation such as $\sigma_{O} r_{(p, q, r), \theta}$ different from the isometric transformations determined before, does not fix the generalized taxicab sphere, so it is not an isometry for the case $a=b \neq c$. Thus, there is no rotary inversion about the origin that preserve the generalized taxicab distance, different from the generalized taxicab isometric transformations determined before.

So, the Euclidean isometries of $\mathbb{R}_{T_{g}}^{3}$ which preserve the generalized taxicab distance for all possible cases are determined. For further studies on this subject, the first question can be "Is there any other transformation which preserve the generalized taxicab distances, which has not to preserve Euclidean distances, in three dimensional space?".

\section{REFERENCES}

[1] Akça, Z. and Kaya, R., On the distance formulae in three dimensional taxicab space, Hadronic Journal, 27, No.5 (2004), 521-532.

[2] Akça, Z. and Kaya, R., On the taxicab trigonometry, Jour. of Inst. of Math. \& Comp. Sci. (Math. Ser.), 10, No.3 (1997), 151-159.

[3] Çolakoğlu H. B., Concerning the alpha distance, Algebras Groups Geom. 8(2011), 1-14.

[4] Ekici, C., Kocayusufoğlu, İ. and Akça, Z., The norm in taxicab geometry, Tr. J. of Mathematics, 22 (1998), 295-307.

[5] Ekmekçi, E., Bayar, A. and Altıntaş, A. K., On the group of isometries of the generalized taxicab plane, International Journal of Contemporary Mathematical Sciences, 10, No.4 (2015), 159-166.

[6] Ekmekçi, S., Akça, Z. and Altıntaş, A. K., On trigonometric functions and norm in the generalized taxicab metric, Mathematical Sciences And Applications E-Notes, 3, No.2 (2015), $27-33$.

[7] Gelişgen, Ö. and Kaya, R., The taxicab space group, Acta Math. Hungar., 122, Issue 1â€"2 (2009), 187â€"200

[8] Gelişgen, Ö. and Kaya, R., On $\alpha$-distance in three dimensional space, Applied Science, 8 (2006), 65-69.

[9] Gelişgen, Ö. and Kaya, R., Generalization of $\alpha$-distance to $n$-dimensional Space, KoG, 10 (2006), 33-35.

[10] Kaya, R., Akça, Z., Günaltılı, İ. and Ö zcan, M., General equation for taxicab conics and their classification, Mitt. Math. Ges. Hamburg, 19 (2000), 135-148.

[11] Kaya, R. and Çolakoğlu, H. B., Taxicab versions of some Euclidean theorems, International Journal of Pure And Applied Mathematics, 26, No.1 (2006), 69-81.

[12] Kocayusufoglu, İ. and Özdamar, E., Isometries of taxicab geometry, Commum. Fac. Sci. Univ. Ank. Series A1, 47 (1998), 73-83.

[13] Krause, E. F., Taxicab Geometry, Addison-Wesley, Menlo Park, California, 1975.

[14] Martin, G. E., Transformation Geometry, Springer-Verlag, New York Inc., 1997, pp.182.

[15] Menger, K., You Will Like Geometry, Guidebook of Illinois Institute of Technology Geometry Exhibit, Museum of Science and Industry, Chicago, Illinois, 1952. 
[16] Schattschneider, D. J., The taxicab group, American Mathematical Monthly, 91, No.7 (1984), 423-428.

[17] Thompson, K. P., The nature of length, area, and volume in taxicab geometry, International Electronic Journal of Geometry, 4, No.2 (2011), 193-207.

[18] Wallen, L. J., Kepler, the taxicab metric, and beyond: An isoperimetric primer, The College Mathematics Journal, 26, No.3 (1995), 178-190.

Current address: Akdeniz University, Vocational School of Technical Sciences, 07070, Konyaaltı, Antalya, TÜRKİYE.

E-mail address: hbcolakoglu@akdeniz.edu.tr

ORCID Address: http://orcid.org/0000-0001-5559-9768 